\title{
Consultation sur le tarif ambulatoire: prochaines étapes?
}

\section{Urs Stoffel}

Dr med., membre du Comité central de la FMH, responsable ad intérim du département Tarifs et conventions pour la médecine ambulatoire en Suisse

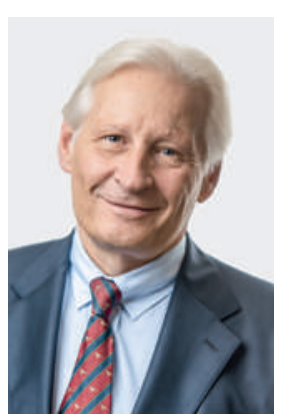

\section{Contexte}

En 2010, la FMH - alors encore seule - a lancé le projet de révision globale de la structure tarifaire ambulatoire sous le nom de «TARVISION». Cette révision a vu le jour suite à la pression engendrée par la publication du rapport du Contrôle fédéral des finances (CDF) sur le TARMED, qui estimait que la structure tarifaire n'était plus appropriée ni conforme aux règles de l'économie d'entreprise et exigeait donc sa révision. A cet effet, l'article 43 alinéa 5 bis de la loi fédérale sur l'assurance-maladie (LAMal) a été complété afin d'octroyer au Conseil fédéral la compétence subsidiaire d'intervenir directement dans la structure tarifaire, mettant ainsi en danger l'autonomie tarifaire des partenaires tarifaires.

Pas moins de 200 personnes réparties dans plus de 25 équipes spécialisées ont collaboré à la révision de la structure tarifaire ambulatoire.

Depuis 2012, H+ et la CTM collaborent étroitement avec la FMH et ont été rejoints en juillet 2015 par curafutura - Les assureurs-maladie innovants. Dans le cadre de cette révision, les partenaires poursuivent tous le même objectif: développer une structure tarifaire conforme à la loi, c'est-à-dire appropriée et conforme aux règles applicables en économie d'entreprise. Au cours des trois dernières années, pas moins de 200 personnes réparties dans plus de 25 équipes spécialisées et épaulées par les experts tarifaires, délégués par les sociétés de discipline médicale, ont collaboré à ce projet et révisé la structure tarifaire ambulatoire chapitre par chapitre.

Le grand défi de la procédure de consultation Le 15 janvier 2016, la FMH a ouvert la procédure de consultation officielle de la structure tarifaire, permettant à chacun de ses membres de donner son avis sur la structure révisée en général, et sur les différentes positions tarifaires en particulier. La structure renseigne uniquement sur la pondération entre les différentes prestations et, à ce titre, vise à garantir l'économicité du système. Pour la LAMal, les négociations sur les prix à proprement parler auront lieu plus tard au niveau cantonal entre les partenaires tarifaires.
Lors de cette consultation, qui a représenté un véritable défi logistique, nous sommes parvenus à mettre à disposition des membres un navigateur tarifaire détaillé, doté d'une interface leur permettant de donner leur avis par voie électronique. Au cours des quatre semaines de la consultation, le navigateur a été téléchargé plus de 15000 fois et plus de 5700 retours nous sont parvenus. C'est l'occasion pour moi d'adresser mes plus vifs remerciements à mon équipe, qui a fourni un travail tout simplement remarquable lors de cette révision et de la phase de consultation. En raison des nombreux retours reçus, le délai de traitement par les équipes spécialisées a été prolongé jusqu’à la mi-mars 2016.

\section{Lancement de la procédure d'approbation interne} à la FMH

Fin mars 2016, le navigateur tarifaire actualisé ainsi que la structure tarifaire définitive, points tarifaires compris, seront publiés sur la plateforme myFMH et pourront donc être consultés librement par tous les membres de la FMH. Cette version sera ensuite soumise pour approbation aux organes décisionnels de la FMH, à savoir l’Assemblée des délégués et la Chambre médicale.

Je souhaite profiter de l'occasion pour remercier tous les membres de la FMH et en particulier les délégués tarifaires des sociétés de discipline.

La Chambre médicale décidera, fin avril 2016, si elle accepte la structure tarifaire ambulatoire révisée. Elle a déjà exprimé le souhait de donner la possibilité aux membres de la FMH de se prononcer, eux aussi, à ce sujet dans le cadre d'une votation générale. Pour autant que la Chambre médicale et les membres de la FMH donnent leur aval, la FMH et ses partenaires pourront soumettre la structure tarifaire au Conseil fédéral à l'été 2016 pour approbation.

Je souhaite profiter de l'occasion pour remercier tous les membres de la FMH, et en particulier les délégués tarifaires des sociétés de discipline, pour leur engagement au cours de la révision de la structure tarifaire ambulatoire, et pour les nombreux retours constructifs lors de la consultation. C'est grâce à votre précieuse contribution que nous pourrons obtenir un tarif médical ambulatoire révisé approprié et équitable. 